\title{
Influence of noodle processing (industrial protocol) on deoxynivalenol.
}

\begin{abstract}
The effects of processing for two types of Asian noodles production (yellow alkaline and instant) on DON levels were investigated. Deoxynivalenol (DON) level at each step of processing was determined using high performance liquid chromatography (HPLC) with a PDA (photodiode array) detector at $218 \mathrm{~nm}$. An acetonitrile: water (17:83 v/v) mixture was used as mobile phase and a MycosepTM \#225 column as clean-up method. Significant reductions of the initial DON levels were found, $43.2 \%$ and $66.6 \%$ in yellow alkaline and instant noodles, respectively. The presence of alkaline salt as an ingredient appeared to be the primary factor influencing the extent of reduction in the two types of noodles. The data indicated that significant reduction of DON have occurred during cooking and frying of both yellow alkaline and instant noodles, respectively. The mechanism of reduction due to cooking is probably a leaching of DON out of the noodle into the cooking medium.
\end{abstract}

Keyword: Deoxynivalenol; Instant noodle; Yellow alkaline noodle. 